Irish Math. Soc. Bulletin

Number 70, Winter 2012, 33-34

ISSN 0791-5578

\title{
GREEN'S FUNCTIONS IN AXIALLY SYMMETRIC SPACE-TIMES WITH APPLICATIONS IN QUANTUM FIELD THEORY AND THE SELF-FORCE
}

\author{
PETER TAYLOR
}

This is an abstract of the $\mathrm{PhD}$ thesis Green's Functions in Axially Symmetric Space-Times with Applications in Quantum Field Theory and the Self-Force written by P. Taylor under the supervision of Prof. A. C. Ottewill at the School of Mathematical Sciences and Complex and Adaptive Systems Laboratory, University College Dublin, Belfield, Dublin 4, and submitted in August 2011.

This thesis is concerned with two important problems in theoretical physics: quantum field theory in black hole space-times and the self-force approach to the study of extreme-mass-ratio inspirals. Physically, these are very different problems, the latter being purely classical while the former involves quantum physics. Mathematically, however, they are similar since they are both most naturally formulated in terms of an appropriate Green's function for the wave equation.

In quantum field theory, the physical quantity of interest is the expectation value of the stress energy tensor operator for the field in some quantum state, which may be defined as the coincidence limit of an appropriate differential operator acting on the Feynman Green's function. This quantity is divergent, even in flat spacetime, and therefore requires renormalization. Renormalization in black hole space-times has a long and successful history but has been very much limited to spherically symmetric cases. The most physically relevant case of the Kerr black hole, which has a reduced axial symmetry, has remained elusive. With this in mind, we consider a simpler axially symmetric case, the Schwarzschild metric

2010 Mathematics Subject Classification. 83C57, 83C45, 83C47, 83C35.

Key words and phrases. Black Holes, Quantum Gravity, Gravitational Radiation.

Received on 7-9-2012.

Support from Irish Research Council for Science, Engineering and Technology through the Embark Initiative Programme is gratefully acknowledged. 
threaded by a cosmic string. On this space-time, we compute the renormalized vacuum polarization on the entire black hole exterior as well as quasi-analytic results for the renormalized stress energy tensor on the black hole horizon. We further discuss the application of this method to the Kerr space-time.

In the self-force problem, one is interested in the classical interaction of a point particle with its own field. Computing the self-force is difficult due to the fact that the field diverges at the particle's location. This divergence arises as a coincidence limit of the retarded Green's function which may be regularized by subtraction of an appropriate singular field. We obtain a completely closedform expression for the retarded Green's function for a static scalar charge in the Kerr space-time as well as an analytic expression for the singular field. We employ these expression to derive analytic results for the self-force on a scalar charge outside the ergosphere of the Kerr black hole. We compare our exact results to the numerical results in the literature. 2 .

(from June 2012) School of Mathematics, Trinity College, Dublin

E-mail address, P. Taylor: ptaylor@maths.tcd.ie 